\title{
Transgenic Approach to Preventing Graft Rejection
}

\author{
Malyshkin AP* \\ Orenburg State Medical Academy, Orenburg, Russia
}

Received: November 15, 2013; Accepted: December 16, 2013; Published: December 19, 2013

*Corresponding author: Malyshkin AP, Orenburg State Medical Academy, Orenburg, Russia, Tel: +7-3532-36-58-20; E-mail: malishkin_54@mail.ru

\begin{abstract}
Organ transplantation entails numerous problems. It is hardly possible to find an absolutely compatible donor, and graft protection through immunosuppression decreases the recipient's resistance to infections. New views on immunity summarized in the concept of linked functions offer a different approach to preventing graft rejection. According to it, genes of the donor's MHC antigens are integrated into the genome of the recipient's hematopoietic stem cells. It is expected that, in this case, the recipient's immune system will recognize the transplant as self and will not reject it.
\end{abstract}

\section{To Editor}

\section{Dear Sir}

Transplantology faces considerable problems with selecting donors and preserving the transplant viability. In most cases, a completely compatible donor is impossible to find, whereas immunosuppressors not only weaken the defense against infections, but also may have toxic effects.

New views on adaptive immunity summarized in the concept of linked functions [1] may serve as a basis of a novel approach to organ and tissue transplantation without selection of the donor or use of immunosuppressors. The concept of linked functions proceeds from the capacity of the immune system for "smart" recognition ensuring the absence of immune response to autoantigens. This results from negative selection of lymphocytes producing antibodies against autoantigens and is, in fact, ultimately determined by the presence of autoantigen genes in the genome. The basic assumption of the suggested approach is that integration of certain MHC I genes of the donor into the genome of the recipient's lymphocyte precursors (lymphocyte or hematopoietic stem cells) before organ or tissue transplantation should preclude the immune response to the graft. In other words, it is assumed that the "smart" recognition mechanisms making transplantation autoantigens "privileged" can be made to act in the same way for the donor's transplantation antigens. It is also conceivable that the mechanism of the formation of tolerance based on "smart" recognition will make it possible to prevent the rejection of not only allogenic, but also xenogenic grafts; this would require integration of the MHC genes of a xenogenic donor to the genome of the recipient's lymphocyte stem cells.

Implementation of this approach to preventing foreign graft rejection would considerably extend the potential of transplantology by eliminating the difficulties related to donor selection and immunosuppression, as well as widening the possibility of using xenogenic transplants, which is hardly possible with conventional methodologies. Apparently, we will be unable to exclude immunosuppressors altogether, but they will be necessary only during presurgical treatment for suppressing the activity of those lymphocytes which have been formed before the gene transplantation.

The specific technique for the integration of the donor's MHC I genes into the recipient's hematopoietic or lymphocyte stem cells has not been developed yet. Undoubtedly, many questions are still to be answered before such a technique becomes practicable; there is a hope, however, that these problems can be solved in the course of further experimental research and development of the new approach to the formation of tolerance to foreign transplantation antigens.

Since the development of the methodology for preventing graft rejection on the basis of this approach will undoubtedly take much effort, I would be grateful to all those who would like to take part in the research or sponsor it.

\section{Acknowledgments}

A.P.M. is grateful to Prof. V.M. Boev, president of Orenburg State Medical Academy; Prof. A.I. Smolyagin; and V.L. Ushakov for their help in the preparation of this paper.

\section{References}

1. Malyshkin, A. P.( 2013). Adaptive Immunity: The Concept of Linked Functions. Immunology Innovation, 1, 1. 